Submitted to Proceedings of Towards Atomic Resolution Anaty

Port Ludlow, Washington, September 6-11, 1998

\title{
Towards Atomic Column-by-Column Spectroscopy
}

\author{
S. J. Pennycook and B. Rafferty
}

Solid State Division, Oak Ridge National Laboratory

P.O. Box 2008, Oak Ridge, Tennessee 37831-6030

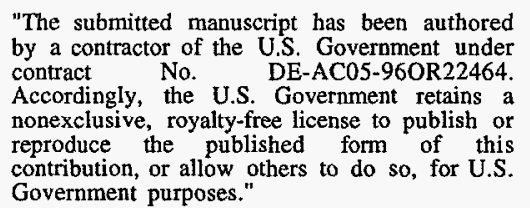

\author{
prepared by \\ SOLID STATE DIVISION \\ OAK RIDGE NATIONAL LABORATORY \\ Managed by \\ LOCKHEED MARTIN ENERGY RESEARCH CORP. \\ under \\ Contract No. DE-AC05-96OR22464 \\ with the \\ U.S. DEPARTMENT OF ENERGY \\ Oak Ridge, Tennessee
}

November 1998 


\section{DISCLAIMER}

This report was prepared as an account of work sponsored by an agency of the United States Government. Neither the United States Government nor any agency thereof, nor any of their employees, make any warranty, express or implied, or assumes any legal liability or responsibility for the accuracy, completeness, or usefulness of any information, apparatus, product, or process disclosed, or represents that its use would not infringe privately owned rights. Reference herein to any specific commercial product, process, or service by trade name, trademark, manufacturer, or otherwise does not necessarily constitute or imply its endorsement, recommendation, or favoring by the United States Government or any agency thereof. The views and opinions of authors expressed herein do not necessarily state or reflect those of the United States Government or any agency thereof. 


\section{DISCLAIMER}

Portions of this document may be illegible in electronic image products. Images are produced from the best available original document. 


\title{
Towards atomic column-by-column spectroscopy.
}

\author{
B Rafferty, S J Pennycook
}

Solid State Division, Oak Ridge National laboratory, PO Box 2008, Oak Ridge TN 37831-6030

\begin{abstract}
The optical arrangement of the scanning transmission electron microscope (STEM) is ideally suited for performing analysis of individual atomic columns in materials. Using the incoherent Z-contrast image as a reference, and arranging incoherent conditions also for the spectroscopy, a precise correspondence is ensured between features in the inelastic image and elastic signals. In this way the exact probe position needed to maximise the inelastic signal from a selected column can be located and monitored during the analysis using the much higher intensity elastic signal. Although object functions for EELS are typically less than $1 \AA$ full width at half maximum, this is still an order of magnitude larger than the corresponding object functions for elastic (or diffuse) scattering used to form the Z-contrast image. Therefore the analysis is performed with an effective probe that is significantly broader than that used for the reference Z-contrast image. For a $2.2 \AA$ probe the effective probe is of the order of $2.5 \AA$, while for a $1.3 \AA$ probe the effective probe is $1.6 \AA$. Such increases in effective probe size can significantly reduce or even eliminate contrast between atomic columns that are visible in the image. However, this is only true if we consider circular collector apertures. Calculations based upon the theory of Maslen and Rossouw (Maslen and Rossouw 1984; Rossouw and Maslen 1984) show that employing an annular aperture can reduce the FWHM of the inelastic object function down to values close $0.1 \AA$. With practical aperture sizes it should be possible to achieve this increased spatial resolution without loosing too much signal.
\end{abstract}




\section{Introduction.}

Internal interfaces are known to dominate the structure-property relationships of many materials and devices. Thus, the ultimate goal of all atomic or near atomic resolution analysis techniques is to determine both the physical and electronic structure of defects, such as a dislocation core or an interface, within a crystalline matrix with atomic column sensitivity. The optical arrangement in the HB603U STEM is ideally suited for performing analysis of individual atomic columns in materials. The major strength of this instrument is that with incoherent Z-contrast imaging it is possible to obtain direct structure images of the atomic configuration of the specimen. One can directly image defects within a sample and determine the physical structure of the sample on-line without having to rely on any post acquisition image processing techniques. Using the incoherent Z-contrast image as a reference, and arranging incoherent conditions for the spectroscopy, a precise correspondence is ensured between features in the inelastic and elastic signals. In this way the exact probe position needed to maximise the inelastic signal from a selected column can be located and monitored during the analysis using the much higher intensity elastic scattering. Electron energy loss spectroscopy (EELS) analysis offers many advantages over X-ray spectroscopy, such as high collection efficiency and the ability to analysis near edge fine structure. Thus, integrating the HB603U with a dedicated McMullan type PEELS system will spawn an instrument with unique performance.

Defining inelastic conditions for the EELS means that the probe can be separated out of the expression for the inelastic intensity, and the real space distribution of scattering power is then referred to as the object function. The inelastic image is then given by the convolution of the probe intensity profile with the object function. As these functions are similar to Gaussians in many cases, 
the best single measure of resolution is the FWHM, as convoluting two Gaussians leads to a Gaussian with FWHM the sum of the two. However, although object functions for EELS are typically less than $1 \AA$ FWHM, this is still an order of magnitude larger than the corresponding object functions for elastic (diffuse) scattering used to form the Z-contrast image. Therefore, the analysis is performed with an effective probe that is significantly broader than that used for the reference Z-contrast image. For a $2.2 \AA$ probe the effective probe is of the order $2.5 \AA$, while for a $1.3 \AA$ probe the effective probe is $1.6 \AA$. Such increases in the effective probe size can significantly reduce or even eliminate the contrast between atomic columns that are visible in the image. A subangstrom probe is thus more essential for atomic resolution analysis than it is for imaging. It may be possible to avoid this larger effective probe size if we could set up the experimental conditions to produce an object function with a smaller FWHM. A possible method to achieve this was indicated by Kohl and Rose (Kohl and Rose 1985) and explicitly outlined by Ritchie and Howie (Ritchie and Howie 1988).

The calculations presented by Kohl and Rose illustrated an increase in the localisation of the inelastic signal with the use of a larger collection aperture, while Ritchie and Howie suggested that the use of an off-axis collector aperture would increase the spatial resolution of the inelastic signal. By removing the low angle inelastic scattering events from the electrons forming the spectrum or inelastic image it should be possible to reduce the size of the effective probe used for the analysis. This can be achieved experimentally by using an annular collector aperture. Here we present calculations of the inelastic object function for K-shell ionisation based upon the theory of Maslen and Rossouw (Maslen and Rossouw 1983; Maslen and Rossouw 1984; Rossouw and Maslen 1984) for both circular and annular collector apertures. We briefly discuss the methodology of this theory, point out some of its limitations and how it could be extended to include effects such as multiple 
scattering. The Z-dependence of the FWHM of the object functions and how this relationship changes with aperture geometry will be presented as will the beam energy dependence of the object functions. The practical limitations to the use of annular apertures such as the physical limitations to the aperture sizes within the HB603U and the potential loss of signal will also be outlined. This last point will also be discussed in the context of high angle plasmon imaging. Firstly we shall outline the ideas of incoherent Z-contrast imaging to show how we can relate the elastic signal to the inelastic signal by the use of an effective probe.

\section{Incoherent Z-contrast imaging.}

It has been shown (Nellist and Pennycook 1998) that by using a large high angle annular detector to collect the elastically scattered electrons a Z-contrast image can be produced which has an implicitly higher spatial resolution than that of a conventional high resolution bright field image. This difference comes from the fact that with the large annular detector in reciprocal space we are summing incoherently over many pairs of overlapping Bragg diffracted discs. Thus, we can separate the integration's over the partial plane-waves forming the probe and that of the wavevector defining the direction of the scattered electron. Fourier transforming back to real space now leaves us with an image which can be written as a convolution

$$
I_{A D F}\left(\mathbf{R}_{0}\right)=\left|P\left(-\mathbf{R}_{0}\right)\right|^{2} \otimes \mid \rho\left(\mathbf{R}_{0}\right)^{2}
$$

where $P\left(\mathbf{R}_{0}\right)$ is the complex amplitude of the probe and $\mid o\left(\left.\mathbf{R}_{0}\right|^{2}\right.$ is the object function of the scattering centre. In contrast to this the intensity in a conventional high resolution image may be written as 


$$
I_{B F}\left(\mathbf{R}_{0}\right)=\mid P\left(-\mathbf{R}_{0}\right) \otimes o\left(\left.\mathbf{R}_{0}\right|^{2}\right.
$$

where $o\left(\mathbf{R}_{0}\right)$ is the inverse Fourier transform of the matrix element describing the scattering event. It can be clearly seen that with incoherent Z-contrast imaging the image interpretation is much more straightforward and the atomic configuration of the sample can be directly imaged. Since the object functions are so narrow $(\leq 0.1 \AA$ ) we can approximate them as being delta functions. Thus, the contrast and resolution in the image is dictated by the probe profile. The optical arrangement of the high resolution STEM allows these incoherent conditions to be established simultaneously for the collection of elastically and inelastically scattered electrons. Consequently the same theory for the incoherent Z-contrast imaging will hold true for that of inelastic imaging. For the inelastic case we have object functions that cannot be approximated as delta functions. The additional width of the object function is now going to smear out the probe profile. We can however interpret this image in a similar fashion to the elastic case as follows. Since the image is given by a convolution, we may replace the object functions by delta functions if we replace the probe profile by an effective probe whose width is larger than the original probe. The effective probe is simply the original convoluted with the real space object function. Although this effective probe may not be much broader than that for the elastic image the slightest increase in width can significantly reduce or even eliminate contrast between the atomic columns that we intend to analyse. To be able to both image and perform EELS analysis on the 1 A scale we need to reduce the size of the effective probe.

\section{Calculating the inelastic object functions.}


Much work has been carried out developing theories to model and calculate the inelastic object function and use them investigate the inelastic imaging process (Maslen and Rossouw 1983; Maslen and Rossouw 1984; Rossouw and Maslen 1984; Allen, McCarthy et al. 1990; Holbrook and Bird 1995; Holbrook 1997). Here we use the theory of Maslen and Rossouw (Maslen and Rossouw 1983; Maslen and Rossouw 1984) as this model provides analytical solutions that are simply evaluated and will be summarised here. As is usual we describe the incident and scattered fast electron as plane waves with wavevectors $\mathbf{k}$ and $\mathbf{k}$ ' respectively. The incident fast electron is scattered from an isolated atom in which the atomic $1 \mathrm{~s}$ electron is ejected from the atom. The momentum of this ejected electron is not fixed by the momentum transferred from the fast electron to the atom, $\hbar\left(\mathbf{k}-\mathbf{k}^{\prime}\right)$, but is distributed over a range of directions that reflect the momentum distribution of the 1s state. A hydrogenic model of the atom is used to provide analytical formulae for the matrix elements. Implicit within the expression for the matrix elements is an integration over all possible directions, $\kappa$, of the ejected atomic electron since this is not detected and we have no knowledge of its momentum. The dipole approximation is not used within this model and so it is valid for all scattering angles of the incident fast electron. Figure 1 shows a schematic of the scattering process including all relevant vectors. In this framework the object function in reciprocal space is (using notation from the Maslen and Rossouw papers) defined as

$$
\tilde{O}(\mathbf{Q})=\int \frac{2|F(1 s, 1 s)| q_{\perp}}{Q_{1}^{2} Q_{2}^{2}} d \theta d \phi
$$

where $\theta$ and $\phi$ define the collector aperture. $F(1 s, 1 s)$ is given by

$$
F(1 s, 1 s)=512 \pi^{3} Z^{5}\left(\frac{\left(Q_{1}^{2}+(Z-\chi)^{2}\right)\left(Q_{2}^{2}+(Z-\chi)^{2}\right)}{\left(Q_{1}^{2}+(Z+\chi)^{2}\right)\left(Q_{2}^{2}+(Z+\chi)^{2}\right)}\right)^{Z / 2 \chi} \frac{H}{P R}
$$

where 


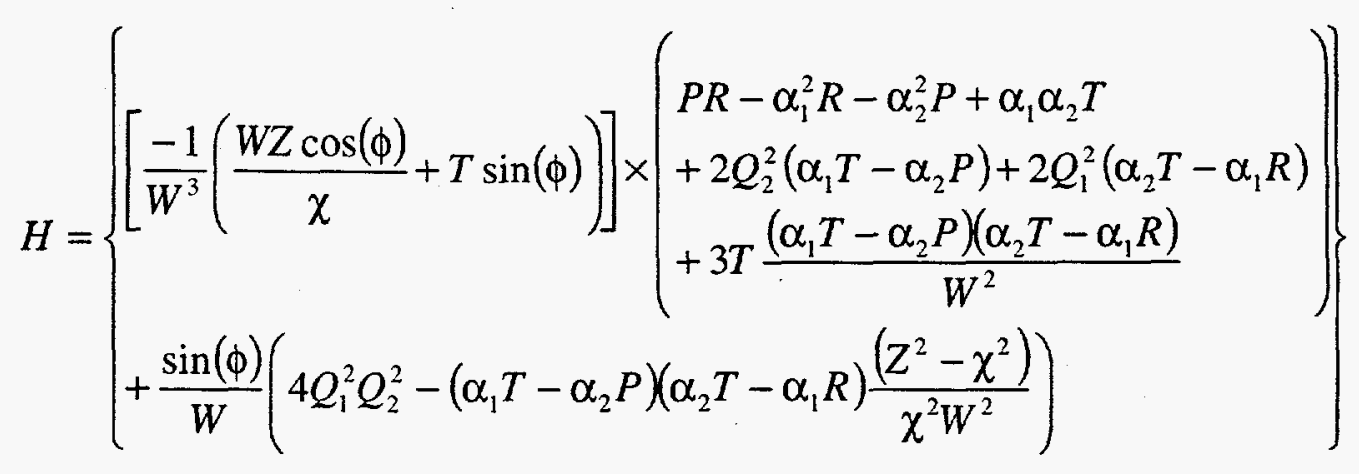

and

$$
\begin{aligned}
& \alpha_{m}=Q_{m}^{2}-\chi^{2}+Z^{2} \\
& P=\alpha_{1}^{2}+4 \chi^{2} Q_{1}^{2} \\
& R=\alpha_{2}^{2}+4 \chi^{2} Q_{2}^{2} \\
& T=\alpha_{1} \alpha_{2}+4 \chi^{2} \mathbf{Q}_{1} \cdot \mathbf{Q}_{2} \\
& W^{2}=P R-T^{2} \\
& \phi=\frac{Z}{\chi} \tan ^{-1}\left(\frac{W}{T}\right) \\
& \mathbf{Q}_{1}=-\frac{1}{2} \mathbf{Q}+\mathbf{q}, \mathbf{Q}_{2}=\frac{1}{2} \mathbf{Q}+\mathbf{q}
\end{aligned}
$$

$\mathbf{Q}$ is a vector lying in the plane of the aperture and $\mathbf{q}=\mathbf{k}^{\prime}-\mathbf{k} \cdot \mathbf{Z}$ is a measure of the screened atomic charge and $\chi=i \kappa$ and an energy of $50 \mathrm{eV}$ has been assigned to the ejected electron above the ionisation threshold. The real space object function $\mid o\left(\left.\mathbf{R}_{0}\right|^{2}\right.$ is the Fourier transform of $\widetilde{O}(\mathbf{Q})$.

There are some obvious limitations to this model such as using hydrogenic wavefunctions. The use of Hartree-Fock wavefunctions would provide a better description of the atomic system and this is work which Oxley and Allen are continuing with at present (Oxley and Allen 1998). It is not yet clear from this work however how the object functions are modified by the use of the HartreeFock wavefunctions. Since we are integrating over all directions of the ejected electron we have assumed a free electron density of states for this electron which, as pointed out by Saldin and Rez (Saldin and Rez 1987), omits any modelling of fine structure effects. Despite these limitations we 
feel that this model should still provide much insight into the effect of aperture geometry on the structure of the inelastic object functions.

\section{Results of simulations.}

\section{Circular apertures.}

Figure 2 shows a plot of the variation of the FWHM of the inelastic object function for different aperture sizes and atomic number. It can be seen that the width of the object function is decreased as the ionisation energy is increased. This is in agreement with the results of Kohl and Rose (Kohl and Rose 1985) where they have the effects of the probe profile as well as the object function. It can also be seen that increasing the size of the aperture for fixed ionisation energy will also reduce the width of the object functions. Figure 3 shows that as the aperture size is increased the large angle scattering contributes more so to the high $\mathrm{Q}$ regions of the object functions in reciprocal space. As was pointed out by Kohl and Rose (Kohl and Rose 1985) this is because we are collecting the electrons that have passed closer to the nucleus and so are contributing the high spatial resolution information to the object function. It can also be seen that this contribution is proportionally larger for lighter atoms. The Õ(Q) falls by 2 and 8 orders of magnitude with a 20 mrad aperture for $\mathrm{Zn}$ and $\mathrm{Be}$ respectively while for a $200 \mathrm{mrad}$ aperture the corresponding figures are 1 and 5 orders of magnitude. It is the exploitation of this property that leads to the use of annular apertures to further reduce the widths of the inelastic object functions. 
Annular apertures.

In Figure 4 we show the oxygen object function in reciprocal space for the individual annuli building up from a 20 to $200 \mathrm{mrad}$ aperture. The contributions from an annular section changes quite drastically as the inner and outer radii are increased by the same amount. The small angle annular sections contribute quite strongly at $Q=0 \AA^{-1}$. As the inner angle is increased the low $Q$ contributions become diminished while the large $\mathrm{Q}$ contributions are strengthened. Consequently the FWHM of the oxygen real space object functions for annuli of $20-40$ and $80-100$ mrad are 0.231 and $0.091 \AA$ respectively. The larger annular sections contain more high resolution information.

A striking result that comes from the use of annular apertures is the change in the Z-dependence of the FWHM of the object functions. It is possible using a sufficiently large inner radii annular aperture to produce an object function for a low $\mathrm{Z}$ atom that has a smaller FWHM than that of a larger $\mathrm{Z}$ atom. The reciprocal space object functions for low $\mathrm{Z}$ atoms have a very narrow distribution and fall off much more quickly than the corresponding high $\mathrm{Z}$ atoms for circular apertures. When the inner angle $\left(\theta_{\text {inner }}\right)$ is increased all contributions with $Q \leq 2 q \cos \left(\theta_{\text {inner }}\right)$ are suppressed. The low $\mathrm{Z}$ atoms being affected the most since their $\mathrm{O}(\mathrm{Q})$ fall off so strongly with $\mathrm{Q}$. Further increasing the inner angle can push the contribution of the low $\mathrm{Q}$ regions of the light atoms below that of heavier atoms [Figure 6] while their high $\mathrm{Q}$ contributions are comparable in value. This reduction of the low $\mathrm{Q}$ parts removes the large tails present in the real space object functions of the light atoms. Thus, the lighter atoms can have a FWHM that is smaller than heavier atoms. 


\section{Beam energy dependence of the object functions.}

Changing the energy of the fast incident electron alters the sizes of the object functions. We see in Figure 7 that, for oxygen K-shell ionisation, increasing the beam energy will decrease the size of the object functions for both circular and annular apertures. According to classical mechanics using a higher energy incident electron should allow the $1 \mathrm{~s}$ atomic electron to be excited from further away (Jackson 1975). The maximum distance from which the electron can excite an electron is proportional to the velocity of the incident electron. Thus, increasing the beam energy by a factor of 3 should surely increase the widths of the object functions. However, increasing the beam energy also means that the incident electron is able to pass much closer to the nucleus of the atom and so this should reduce the widths of the object functions. If these variations in the range of possible impact parameters are weighted with the corresponding probability of producing the required excitation then it is likely that the overall effect is to reduce the size of the average impact parameter with increased beam energy.

\section{Possible limitations to the use of annular apertures.}

We have seen in these calculations that it is possible in principle to reduce the FWHM of the object functions down to values close to $0.1 \AA$. However, this was only possible by using annular collector apertures with outer radii approaching $200 \mathrm{mrad}$ ! If we are to use the incoherent Z-contrast image as a reference during the acquisition of these spectra the choice of aperture size will be dictated by the inner angle of the annular dark field detector (approx. $30-40 \mathrm{mrad}$ ). This means that

for say oxygen we can reduce the width of the object function from $0.33 \AA$ to $0.23 \AA$. Although this 
is not as striking as that which could be achieved with larger annular apertures this may be enough to allow one to distinguish between two columns of oxygen that are separated by only $1.5 \AA$. This would still be quite an achievement!

The use of an annular aperture means that we cut out the low angle scattering from the spectrum. This may mean that we do not have enough signal to collect to fully exploit the advantage of the annular apertures. However, Figure 8 shows oxygen object functions for a circular aperture and the proposed annular apertures. The peak intensity for the annular apertures is only reduced slightly from that of the circular aperture. As far as imaging is concerned we will not lose too much signal per pixel, but for spectroscopy we should consider the integrated signal that these object functions correspond to. Although this is true for the total signal it is not so for column-by-column EELS. We will lose some signal but this will not that large an amount and acquisition of spectra with these apertures should still be possible.

If it were possible to use the much larger annular apertures and the low signal was not a limiting factor would we be able to get the spatial resolution that the calculations would imply. The problem that we would encounter would be that of multiple scattering effects. This is not included within these calculations. If we compare the probability of a single inelastic scattering event to a large angle ( $>40 \mathrm{mrad}$ ) and that of a single low angle inelastic event followed by a high angle elastic event, or visa versa, we would find that the second scenario is more probable. We would still obtain a high spatial resolution but this would be because of the elastic scattering contribution. This will probably have some significant implications on the interpretation of atomic resolution plasmon imaging at surfaces (Muller and Silcox 1995). In our case we can simply place the probe in specific locations within the unit cell to measure the inelastic image contrast. Comparing this with the elastic 
image contrast we will be able to accurately quantify the loss of contrast due to delocalisation (i.e. the effective probe broadening).

\section{Conclusions.}

We have briefly outlined the theory of Maslen and Rossouw and mentioned some of its limitations. Using their approach, we have calculated the inelastic object functions for both circular and annular collector apertures. The effects of the size of the inner angle of the annular apertures, the Z-dependence and the beam energy dependence on the FWHM of the object functions have been discussed. It was shown that it is possible that with annular apertures it is possible for the lighter $\mathrm{Z}$ atoms to have a real space object with a smaller spatial extent than a larger $\mathrm{Z}$ atom. This result was explained by the greater proportional suppression of the low $Q$ regions of the reciprocal space object functions. Contrary to classical impact parameters, increasing the beam energy actually reduces the widths of the real space object functions due to the incident electron being able to approach the nucleus much more closely. The limitations and practicalities of the use of annular apertures have been pointed out and the possible effects of multiple scattering were discussed. Experiments are necessary to see if annular apertures are useful in practice for improving spatial resolution in EELS.

\section{Acknowledgements.}

The authors would like to thank P D Nellist for fruitful discussions related to this work. This research was supported by Lockheed Martin Energy Research Corp. under DOE Contract No. 
DE-AC05-96OR22464, and by appointments to the ORNL Postdoctoral Research Associates Program administered jointly by ORNL and ORISE. 


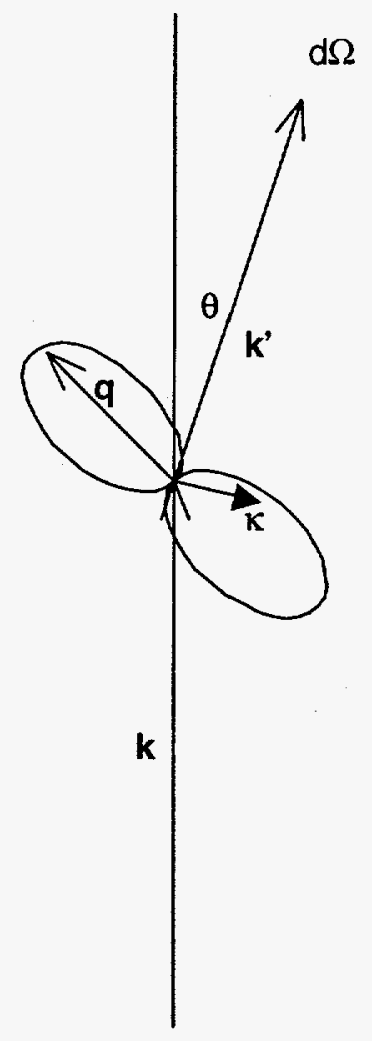

Figure 1: Schematic diagram showing the inelastic scattering process. 


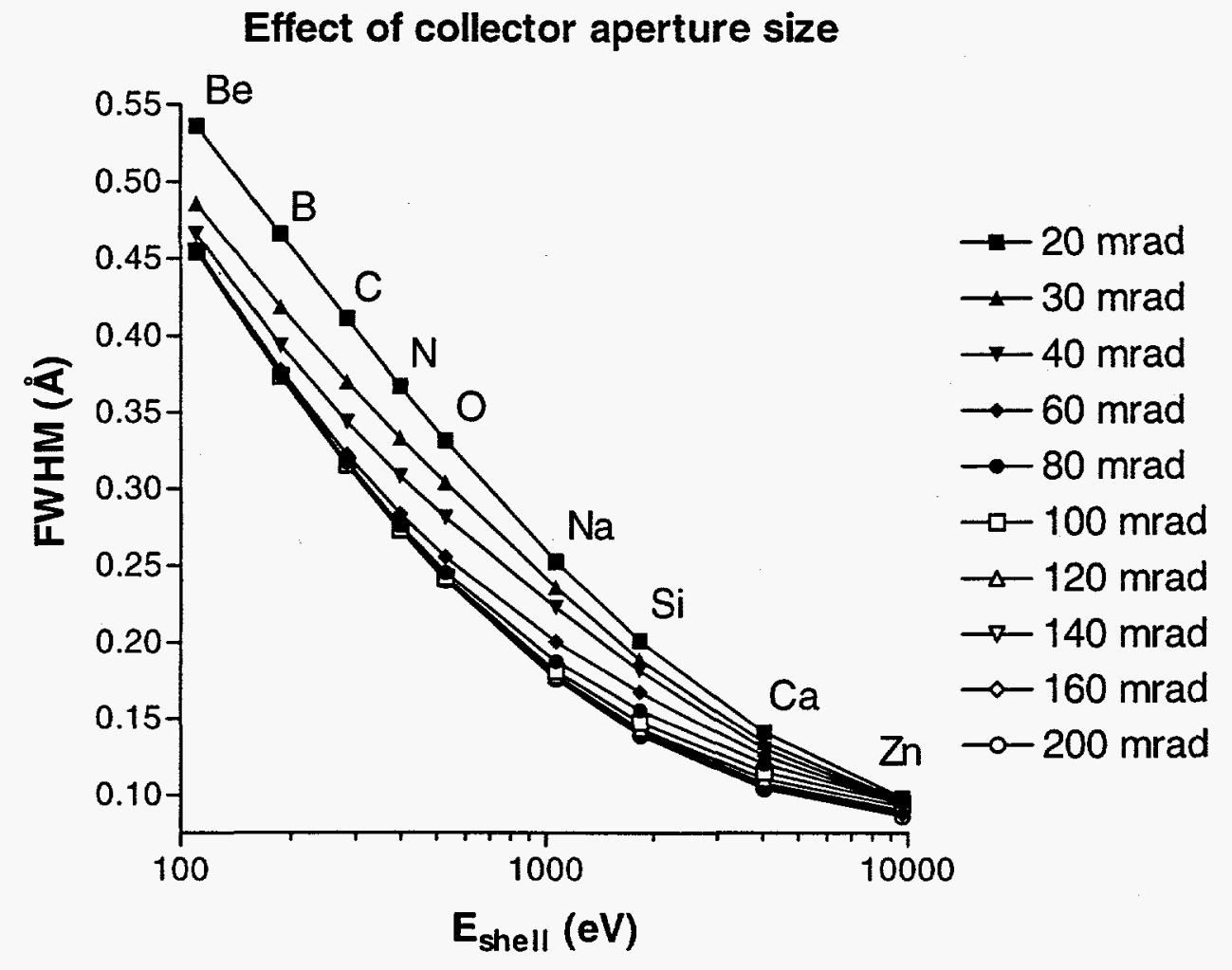

Figure 2: Plot to show the variation of the FWHM of the inelastic object functions for different aperture sizes and atomic number. 
$\mathrm{Be}$

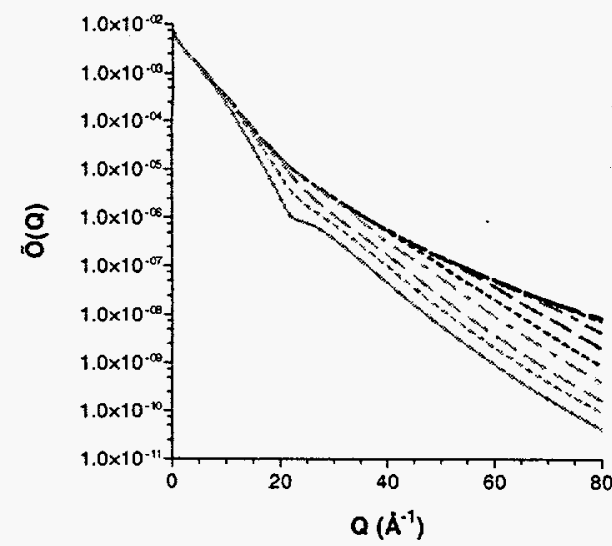

$\mathrm{Ca}$

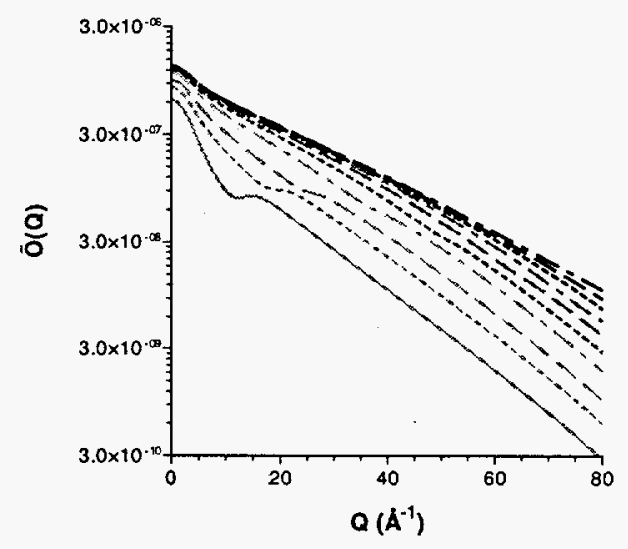

$-20 \mathrm{mrad}$

-.. $30 \mathrm{mrad}$

$--40 \mathrm{mrad}$

$-.60 \mathrm{mrad}$

..- $80 \mathrm{mrad}$

$--100 \mathrm{mrad}$

$-.120 \mathrm{mrad}$

-.- $140 \mathrm{mrad}$

$--160 \mathrm{mrad}$

$-.200 \mathrm{mrad}$

$-20 \mathrm{mrad}$

-. $30 \mathrm{mrad}$

$--40 \mathrm{mrad}$

- -60 mrad

..- $80 \mathrm{mrad}$

$--100 \mathrm{mrad}$

$-.120 \mathrm{mrad}$

..- $140 \mathrm{mrad}$

$--160 \mathrm{mrad}$

$-.200 \mathrm{mrad}$

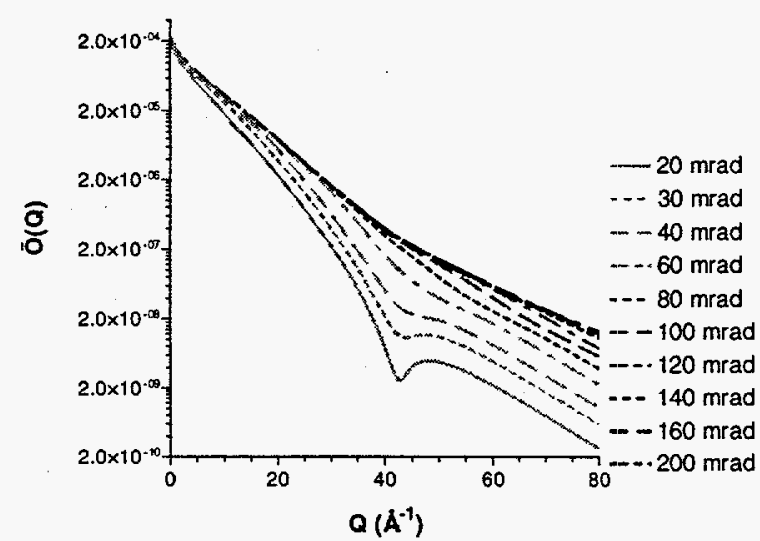

Zn

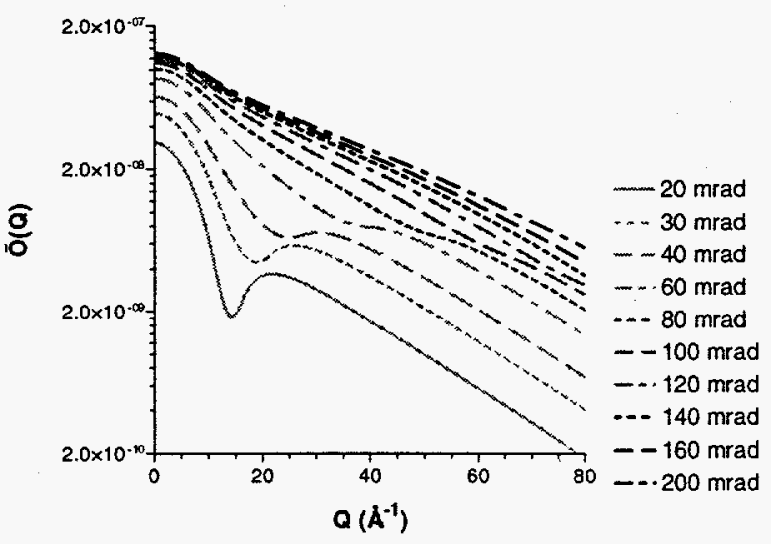

Figure 3: Plots of the object functions in reciprocal space showing how the increased aperture sizes increase the contributions to the larger $Q$ regions. This additional contribution is proportionally larger for the lighter atoms. 


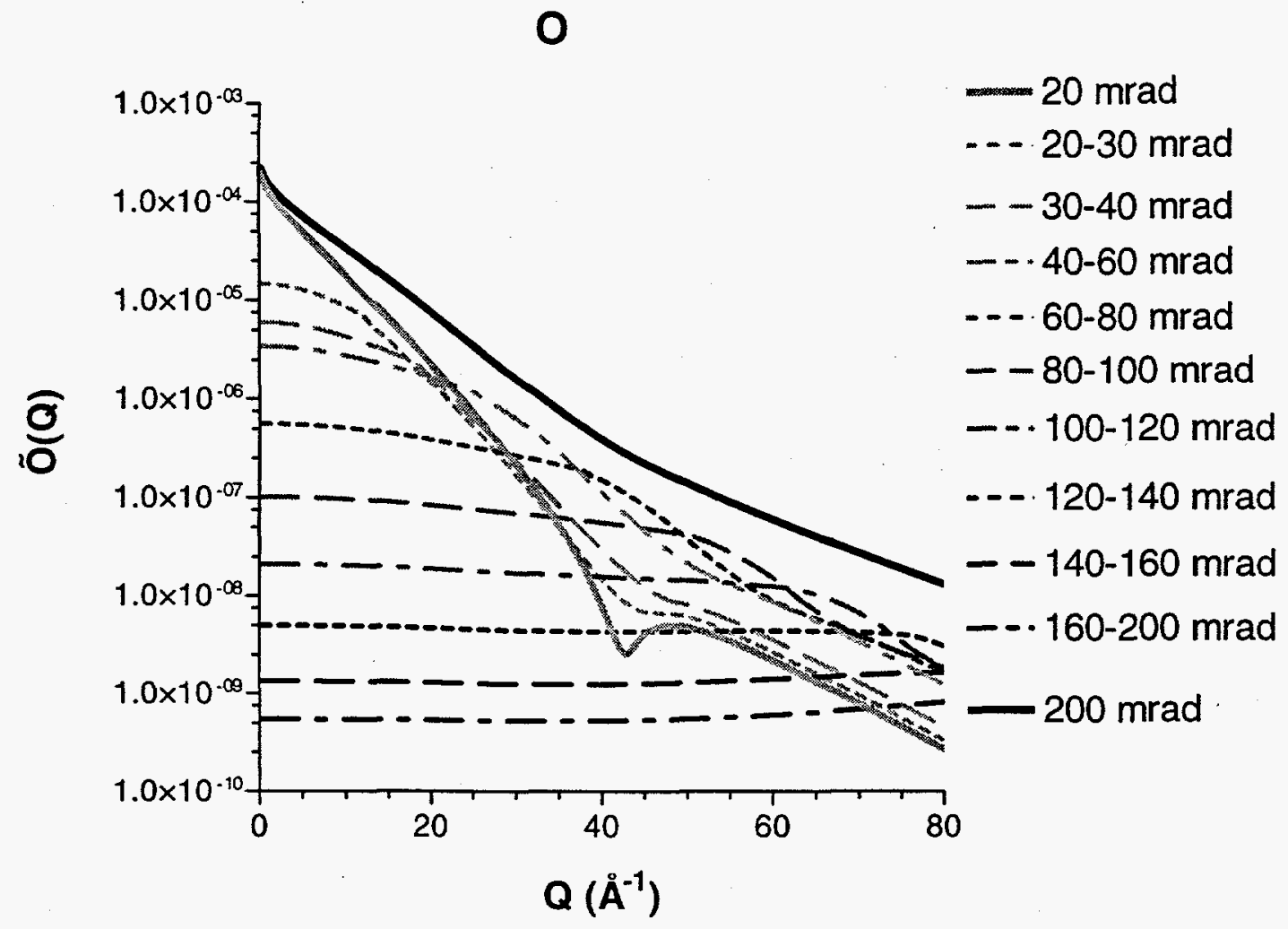

Figure 4: The oxygen object function in reciprocal space showing the contributions from the different annuli as we increase from 20 to $200 \mathrm{mrad}$. 

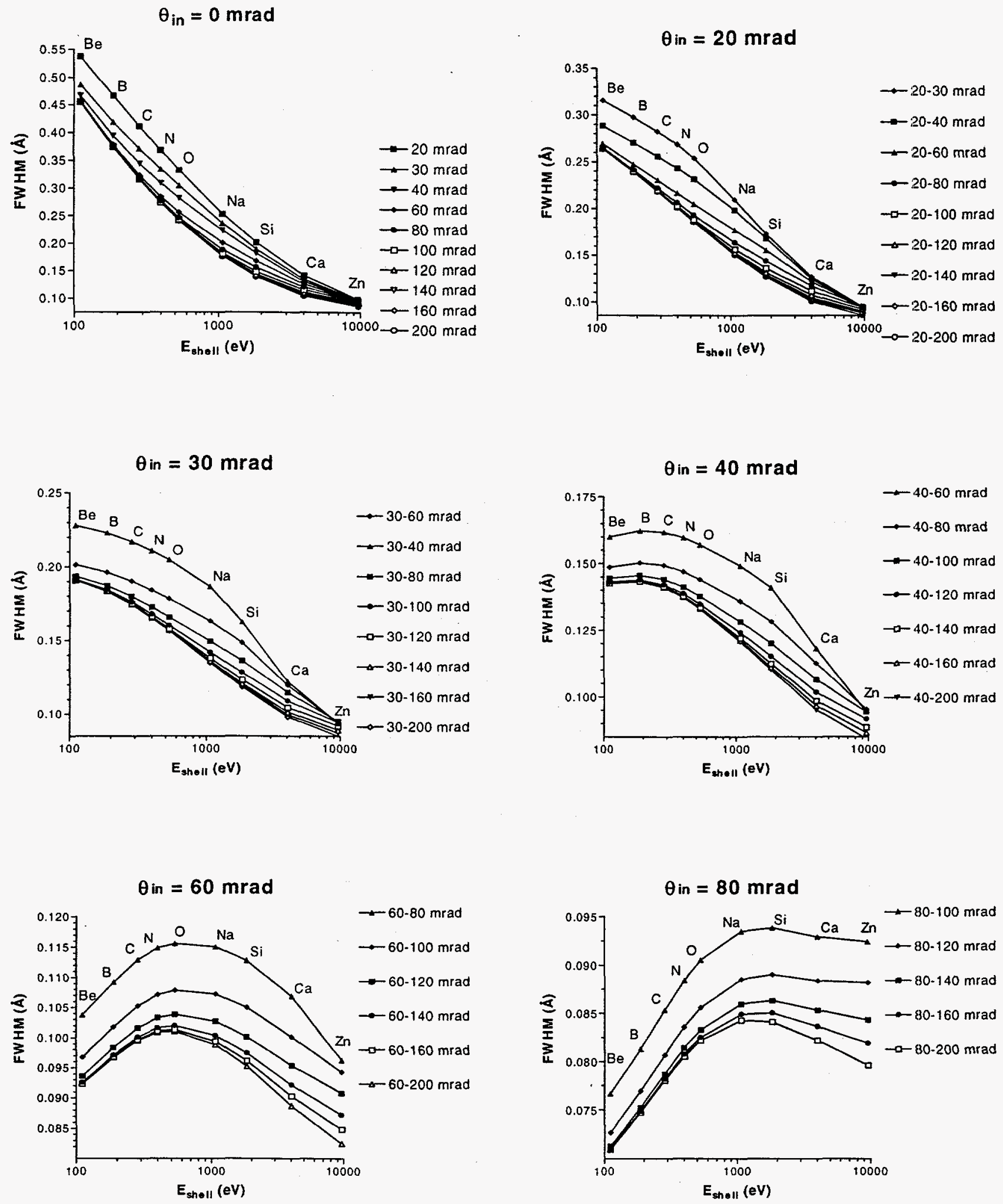

Figure 5: Plots to show the variation in the Z-dependence of the FWHM with the use of different sized annular collector apertures. 
$100 \mathrm{mrad}$
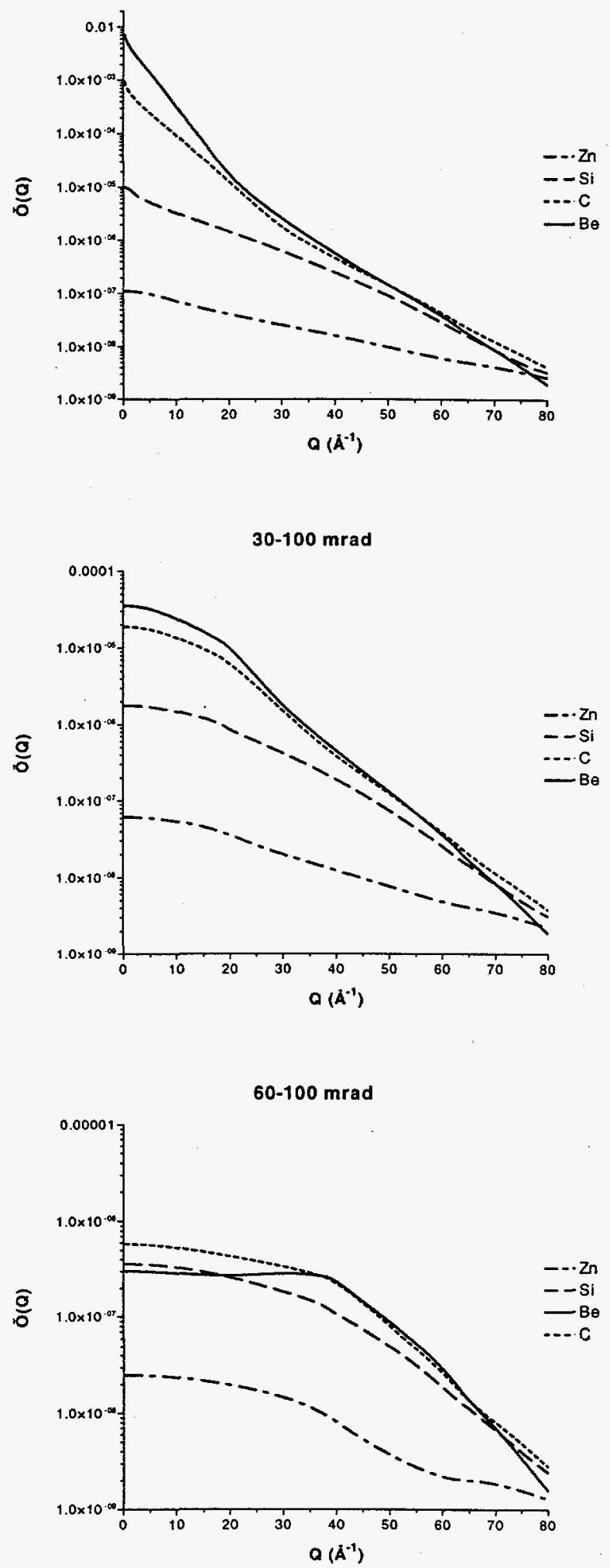
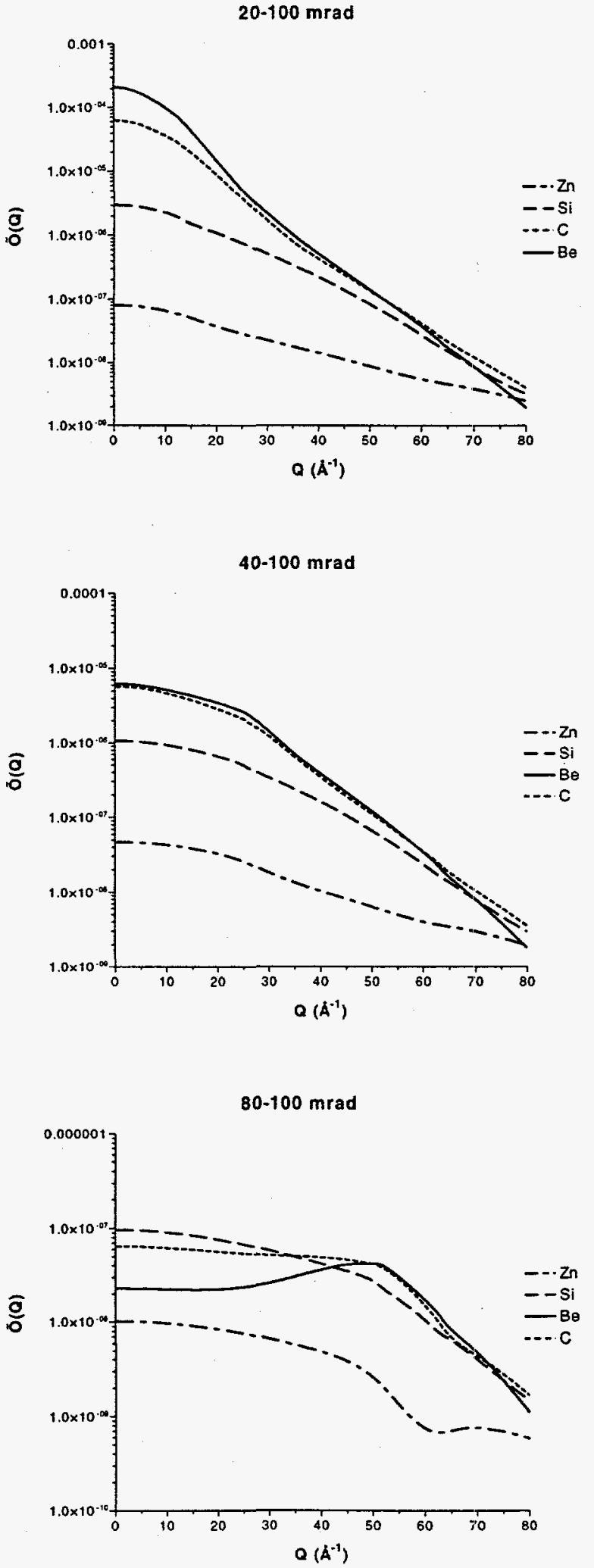

Figure 6: reciprocal space object functions. Increasing the inner angle suppresses the low $Q$ regions for light atoms more so than for heavier atoms. 

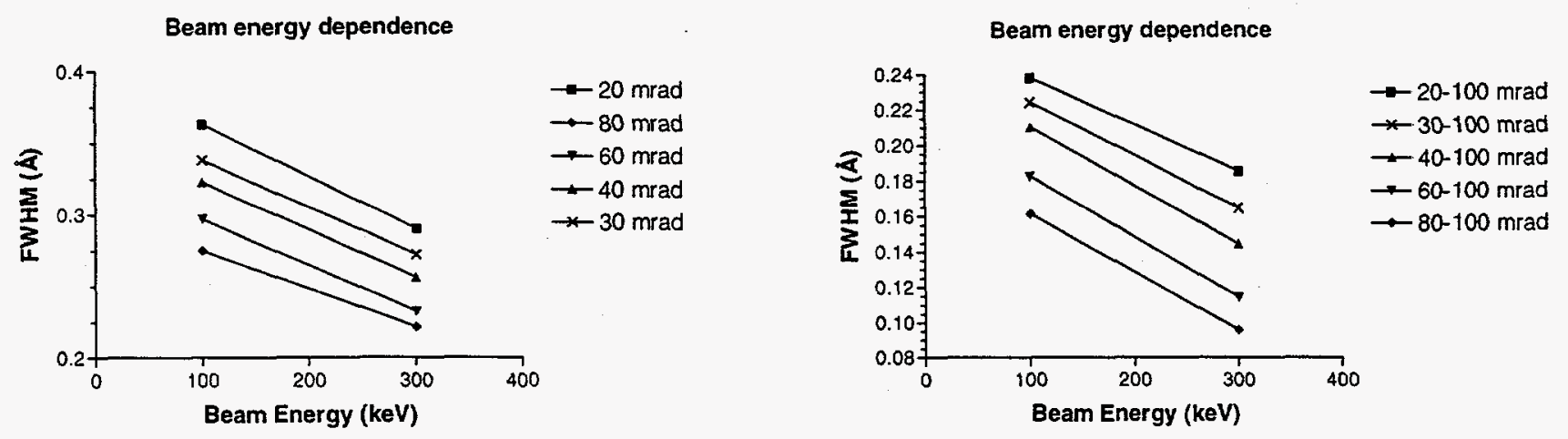

Figure 7: Beam energy dependence on the object functions of oxygen.

\section{Oxygen object functions.}

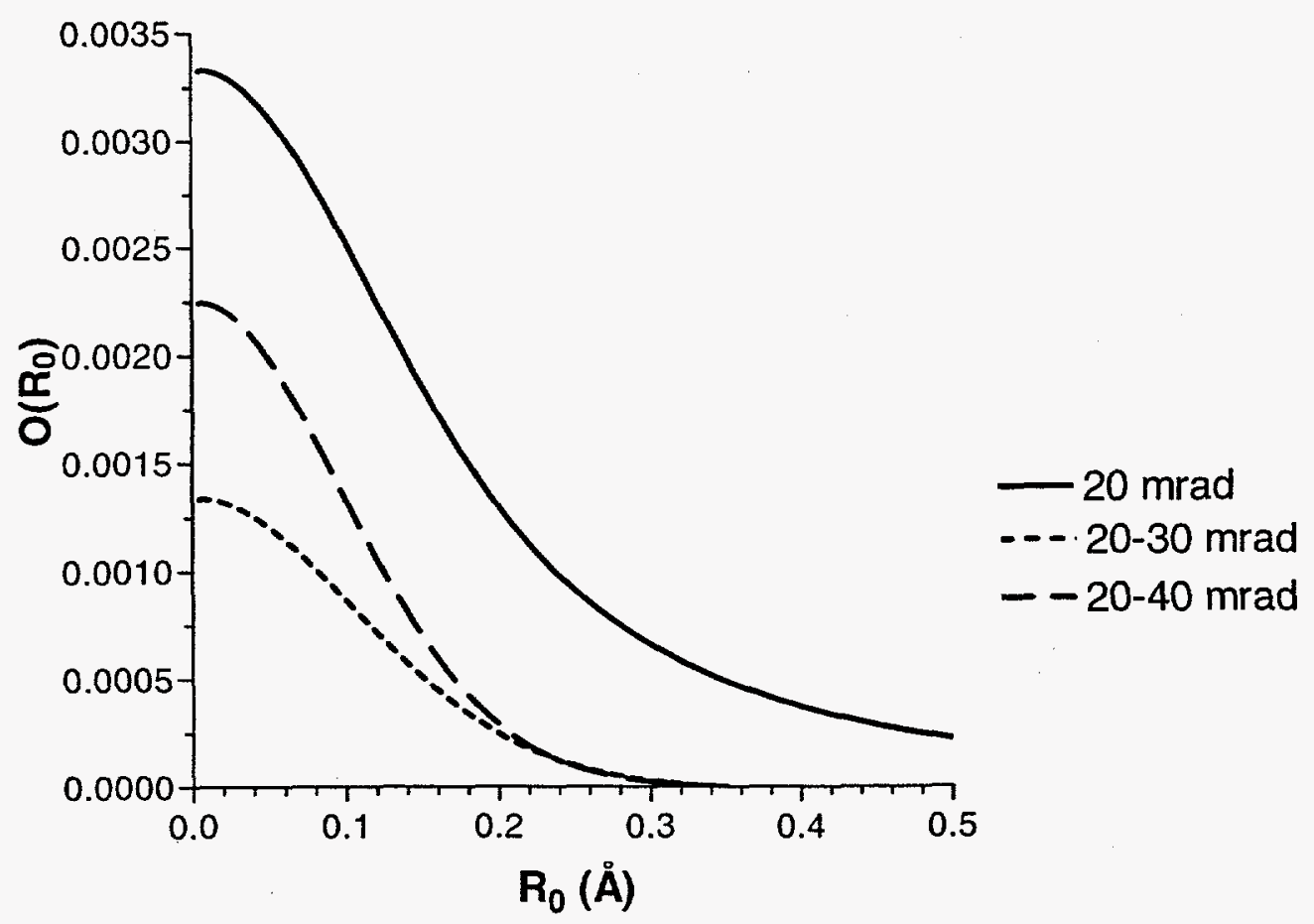

Figure 8: Object functions for oxygen $K$-shell. The use of the annular aperture does not reduce the peak intensity too drastically from that for the circular aperture. 


\section{References}

Allen, L. J., I. E. McCarthy, et al. (1990). "Effects of Diffraction On the (E, 2e) Reaction in Crystals." Australian Journal of Physics 43(4-5): 453-464.

Holbrook, O. (1997). Diffraction effects in inelastic imaging? Physics. Bath, University of Bath.

Holbrook, O. F. and D. M. Bird (1995). "Theoretical Modelling of Atomic Images Formed With Inelastically Scattered Electrons." Institute of Physics Conference Series 147: 175-178.

Jackson, J. D. (1975). Classical Electrodynamics. New York, Chichester, Brisbane, Toronto, Singapore, J Wiley \& Sons.

Kohl, H. and H. Rose (1985). Theory of image formation by inelastically scattered electrons in the electron microscope.

Maslen, V. W. and C. J. Rossouw (1983). "The Inelastic-Scattering Matrix Element and Its Application to Electron-Energy Loss Spectroscopy." Philosophical Magazine a-Defects and Mechanical Properties 47(1): 119-130.

Maslen, V. W. and C. J. Rossouw (1984). "Implications of (e,2e) Scattering For Inelastic ElectronDiffraction in Crystals .1. Theoretical." Philosophical Magazine a-Defects and Mechanical Properties 49(6): 735-742.

Muller, D. and J. Silcox (1995). "Delocalisation in inelastic scattering." Ultramicroscopy 59: 195213.

Nellist, P. D. and S. J. Pennycook (1998). "Accurate structure determination from image reconstruction in ADF STEM." Journal of Microscopy-Oxford 190(Pt1-2): 159-170.

Oxley and L. Allen (1998). Private communication.

Ritchie, R. H. and A. Howie (1988). "Inelastic-Scattering Probabilities in Scanning-Transmission Electron- Microscopy." Philosophical Magazine a-Physics of Condensed Matter Defects and Mechanical Properties 58(5): 753-767. 
Rossouw, C. J. and V. M. Maslen (1984). "Implications of (e, 2e) Scattering For Inelastic ElectronDiffraction in Crystals .2. Application of the Theory." Philosophical Magazine a-Defects and Mechanical Properties 49(6): 743-757.

Saldin, D. K. and P. Rez (1987). "The theory of the excitation of atomic inner-shells in crystals by fast electrons." Phil. Mag. B 55: 481-489. 